\title{
INFLUÊNCIA DA ARQUITETURA NA ESTIMATIVA DE VOLUME DE ÁRVORES INDIVIDUAIS POR MEIO DE REDES NEURAIS ARTIFICIAIS ${ }^{1}$
}

Eric Bastos Gorgens², Helio Garcia Leite ${ }^{3}$, José Marinaldo Gleriani ${ }^{3}$, Carlos Pedro Boechat Soares ${ }^{3}$ e Aline Ceolin $^{4}$

\begin{abstract}
RESUMO - As redes neurais supervisionadas são compostas por unidades de processamento organizadas de forma paralela, em que cada uma delas computa determinadas funções matemáticas. As unidades são organizadas em camadas e ligadas por pesos sinápticos que ponderam as entradas, buscando ajustá-los a um padrão de saída previamente estabelecido. É fundamental a correta definição do número de camadas e da quantidade de neurônios em cada uma delas, uma vez que o treinamento é influenciado diretamente por esses parâmetros. Para explorar esse ponto, dados de cubagem de cinco empresas diferentes foram reunidos em uma planilha e, de forma aleatória, divididos em conjunto de treinamento e conjunto de validação. Os dados foram apresentados para três redes com arquiteturas diferentes. A avaliação foi feita por meio de gráficos de resíduos e teste $\mathrm{t}(\mathrm{p}<=0,05)$. Com base nos resultados, foi possível concluir que, para obter estimativas de volume por árvore, a rede neural deve ser construída com mais de 10 neurônios na primeira camada, sendo recomendado o uso de mais de uma camada intermediária.
\end{abstract}

Palavras-chave: Cubagem; Neurônios; Camada.

\section{INFLUENCE OF THE ARCHITECTURE IN ESTIMATED VOLUME OF INDIVIDUAL TREES USING ARTIFICIAL NEURAL NETWORKS}

\begin{abstract}
Supervised neural networks are composed of parallel processing units. Each unit, called neurons, computes certain mathematical functions. The units are arranged in layers and connected by synaptic weights to balance the entries, trying to adjust them to a predetermined output pattern. The correct definition of the number of layers and the number of neurons in each layer are crucial, once the training is directly influenced by these parameters. To explore this point, data of scaling from five different regions were arranged in a spreadsheet and randomly divided into training and validation set. Data were presented for three networks with different architectures. The evaluation was performed using residual plots and t test $(p<0.05)$. To estimate volume per tree, the neural network must be built with more than 10 neurons in the first layer, and it is recommended the use of more than one intermediate layer.
\end{abstract}

Keywords: Tree scaling; Neurons; Layers.

\footnotetext{
${ }^{1}$ Recebido em 08.02.2011, aceito para publicação em 27.02.2014.

${ }^{2}$ Departamento de Ciências Florestais, Escola Superior de Agricultura 'Luiz de Queiroz', USP, Brasil. Email: < gorgens@usp.br>.

${ }^{3}$ Departamento de Engenharia Florestal, Universidade Federal de Viçosa, UFV, Brasil. E-mail: <hgleite@gmail.com>, <gleriani@ufv.br> e<csoares@ufv.br>.

${ }^{4}$ Departamento de Engenharia Florestal, Universidade Federal de Santa Maria, UFSM. Email: <aline_ceolin@hotmail.com>.
} 


\section{INTRODUÇÃO}

Nas Ciências Agrárias, a técnica de redes neurais do tipo associativa já é amplamente utilizada no sensoriamento remoto, como pode ser visto em trabalhos de Gimblett e Ball (1995), Kavzoglu e Mather (2003) e Haykin (2001). No entanto, trabalhos com redes do tipo preditiva ainda vêm sendo pouco explorados, especialmente na área de Mensuração e Manejo Florestal que demanda soluções deste tipo. Nessa área, a predição se dá em diversos níveis, de árvores individuais a povoamentos; e em diferentes características, imprescindíveis ao manejo, como volume, perfil de tronco, distribuição de altura e diâmetro, perfil da casca e, até mesmo, perfil do cerne.

A estimativa do volume pode ser feita utilizando modelos volumétricos (SHARMA; ODERWALD, 2001), modelos de múltiplos volumes (SILVA et al., 2007) e modelos de afilamento, também conhecidos como modelos de Taper (BI; LONG, 2001; GARBER; MAGUIRE, 2003) ou modelos de razão (CAMPOS; LEITE, 2006). Quando se pretende estimar um único tipo de volume por árvore, são utilizados modelos volumétricos (DIAMANTOPOULOU, 2005).

Ao longo dos anos, as empresas acumulam grande quantidade de dados de cubagem, incluindo árvores com diferentes características e variações de forma (LEITE et al., 1995). Esses dados podem ser utilizados para implementar um projeto de redes neurais, cujo objetivo final é a estimativa do volume de árvores sem o uso de equações volumétricas e de dados específicos de cubagem (GORGENS et al., 2009).

A predição pode ser ainda mais complexa, quando se leva em consideração que os plantios ocorrem em grandes extensões de terras não contínuas e com diferentes materiais genéticos que podem ser de clones e, até mesmo, espécies diferentes. Isso implica existência de diferentes “combinações” genéticas e edafoclimáticas, que influenciam diretamente nas características a serem estimadas.

A técnica de redes neurais caracteriza-se por possuir muitos parâmetros, que em sua maioria são definidos de forma empírica. Essas características podem ser divididas em dois grupos: características associadas à arquitetura e características associadas à heurística de aprendizagem.
A primeira decisão que o usuário deve tomar é quanto à arquitetura, pois a partir dela se definem as características de aprendizagem. As principais características associadas à arquitetura são: o número de camadas intermediárias, o número de neurônios dessas camadas, o sentido e direção das sinapses e a função de ativação dos neurônios (KAVZOGLU; MATHER, 2003).

Sendo uma rede do tipo feed forward, o principal algoritmo de treinamento é o da retropropagação do erro, que comumente se apresenta acrescida do termo momentum. Esse tipo de rede possui as ligações entre os neurônios, ocorrendo sempre no sentido entradasaída; associado a cada uma dessas ligações, existe um valor denominado peso. A eficiência do aprendizado está diretamente relacionada aos parâmetros do algoritmo de treinamento, do número de neurônios e do número de camadas (GORGENS et al., 2006).

O objetivo deste estudo foi avaliar três arquiteturas diferentes com diversas quantidades de neurônios e de camadas intermediárias.

\section{MATERIAL E MÉTODOS}

Os dados de cubagem de eucalipto (Eucalyptus spp.) foram obtidos de quatro empresas florestais, localizadas na Bahia, São Paulo e em Minas Gerais. Já os de teca foram obtidos de uma quinta empresa, localizada no Mato Grosso. As coletas foram realizadas no ano 2001, em povoamentos com idades entre 4 e 6 anos. Para cada uma dessas empresas, denominadas por local 1 a local 5, obtiveram-se o diâmetro a 1,30 de altura (dap), a altura total ( $h t$ ) e o volume por árvore cubada, interando, assim, informações de 988 árvores (Tabela 1).

As árvores cubadas foram reunidas em uma única planilha eletrônica. Para diferenciar os locais de coleta, cinco variáveis dummy foram criadas, de forma que, quando o local era igual a 1, a variável dummy 1 assumia valor 1; nos demais locais, dummy 1 assumia valor 0 . A mesma regra vale para as outras variáveis dummy.

Os inputs da rede foram gerados ao multiplicar dap e ht pelas variáveis dummy. Dessa forma, foram obtidas 10 variáveis de entrada, sendo cinco delas referentes a dap e cinco a $h t$. 
Tabela 1 - Análise descritiva dos dados de cada local. Table 1 - Descriptive analysis of data from each site.

\begin{tabular}{ccccc}
\hline Local & Estatística & dap $(\mathrm{cm})$ & alt $(\mathrm{m})$ & volume $\left(\mathrm{m}^{3}\right)$ \\
\hline SP & Média & 9,7155 & 9,0860 & 0,0380 \\
& Desvio-padrão & 2,5106 & 2,0043 & 0,0223 \\
& Mínimo & 3,0000 & 3,4000 & 0,0015 \\
& Máximo & 15,6000 & 13,4000 & 0,1094 \\
\hline BA & Média & 18,0521 & 0,3413 \\
& Desvio-padrão & 6,4886 & 24,0611 & 0,2495 \\
& Mínimo & 7,0000 & 0,0231 \\
& Máximo & 31,0000 & 11,5000 & 1,0093 \\
\hline MG1 & Média & 24,6673 & 35,0000 & 0,5664 \\
& Desvio-padrão & 5,5074 & 0,3098 \\
& Mínimo & 16,5000 & 0,1472 \\
& Máximo & 34,4000 & $1,21,2127$ & 1,1093 \\
\hline MG2 & Média & 18,1527 & 0,4126 \\
& Desvio-padrão & 6,2939 & 36,3000 & 0,3719 \\
& Mínimo & 8,6500 & 0,0476 \\
& Máximo & 37,7500 & 6,1370 & 2,3597 \\
\hline MT & Média & 9,8667 & 15,7800 & 0,0344 \\
& Desvio-padrão & 2,8660 & 47,6100 & 0,0195 \\
& Mínimo & 5,7000 & 8,0633 & 0,0108 \\
& Máximo & 15,9000 & 1,9049 & 0,0766 \\
\hline
\end{tabular}

Embora houvesse espécies diferentes, não foi necessário atribuir variável para elas, pois o local já indicava indiretamente a espécie plantada. Caso essa diferenciação não ocorresse, uma variável categórica deveria ser acrescentada.

Depois de estruturado o banco de dados, estes foram normalizados e equalizados. A normalização evita a polarização da rede e a equalização dos dados, a saturação das funções de ativação.

Para treinamento e validação, os dados foram separados de forma aleatória em dois conjuntos. $\mathrm{O}$ de treinamento, composto por $60 \%$ dos dados, e o de validação, composto pelos dados complementares (40\%).

Foram construídas redes com três arquiteturas diferentes (Figura 1), todas usando a função de ativação logística. Os pesos iniciais foram gerados de forma aleatória entre os valores -0,5 e 0,5. As camadas foram interligadas por sinapses completas, ou seja, cada neurônio da camada i foi ligado a todos os neurônios da camada seguinte $(i+1)$, sendo as sinapses orientadas somente para frente (tipo feed forward).

O algoritmo de aprendizado utilizado foi o da retropropagação do erro (back propagation) e os parâmetros de treinamento, taxa de aprendizagem de
0,2 e termo momentum de 0,9. O software utilizado para o treinamento foi o Java SNNS (ZELL et al., 1998) (disponível em: <http://www-ra.informatik.unituebingen.de/SNNS $>$ ). O treinamento foi encerrado quando o gráfico de resíduos tendia à estabilização.

As redes foram avaliadas quanto à eficiência, por meio do teste $\mathrm{t}$, comparando valores estimados e valores observados no conjunto de validação. As redes que resultaram em diferenças não significativas, em relação aos valores observados, foram analisadas quanto à distribuição dos resíduos. Os dados processados pela rede foram submetidos à operação inversa do préprocessamento, visando restaurar a escala original dos dados.

\section{RESULTADOS}

Após o treinamento, foram obtidas três redes, baseadas nas três arquiteturas propostas. Os dados de validação foram, então, processados e comparados aos valores observados pelo teste t. Para o nível de significância de 5\%, as redes 42, 46 e 50 de arquiteturas 1 , 2 e 3, respectivamente, e com a função de ativação logística, apresentaram valores estimados estatisticamente iguais aos observados. Essas redes, então, foram avaliadas quanto à distribuição dos resíduos (Figura 2).

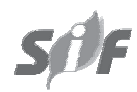

Revista Árvore, Viçosa-MG, v.38, n.2, p.289-295, 2014 


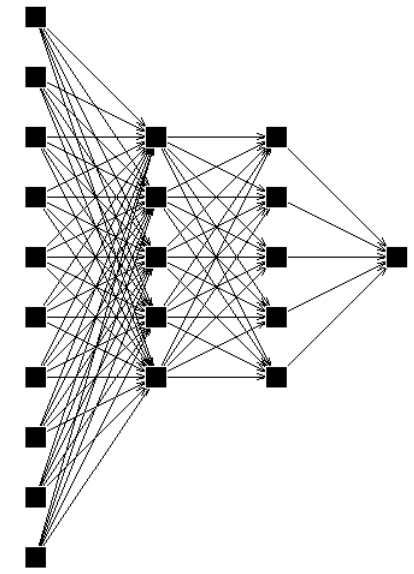

(a) Arquitetura 1

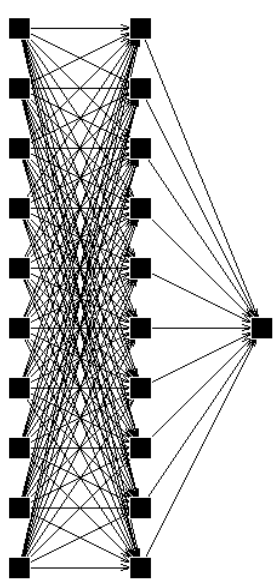

(b) Arquitetura 2

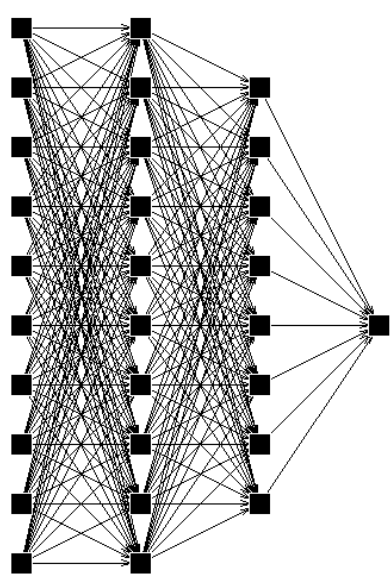

(c) Arquitetura 3

Figura 1 - Arquiteturas das redes utilizadas. Figure 1 - Network layout.
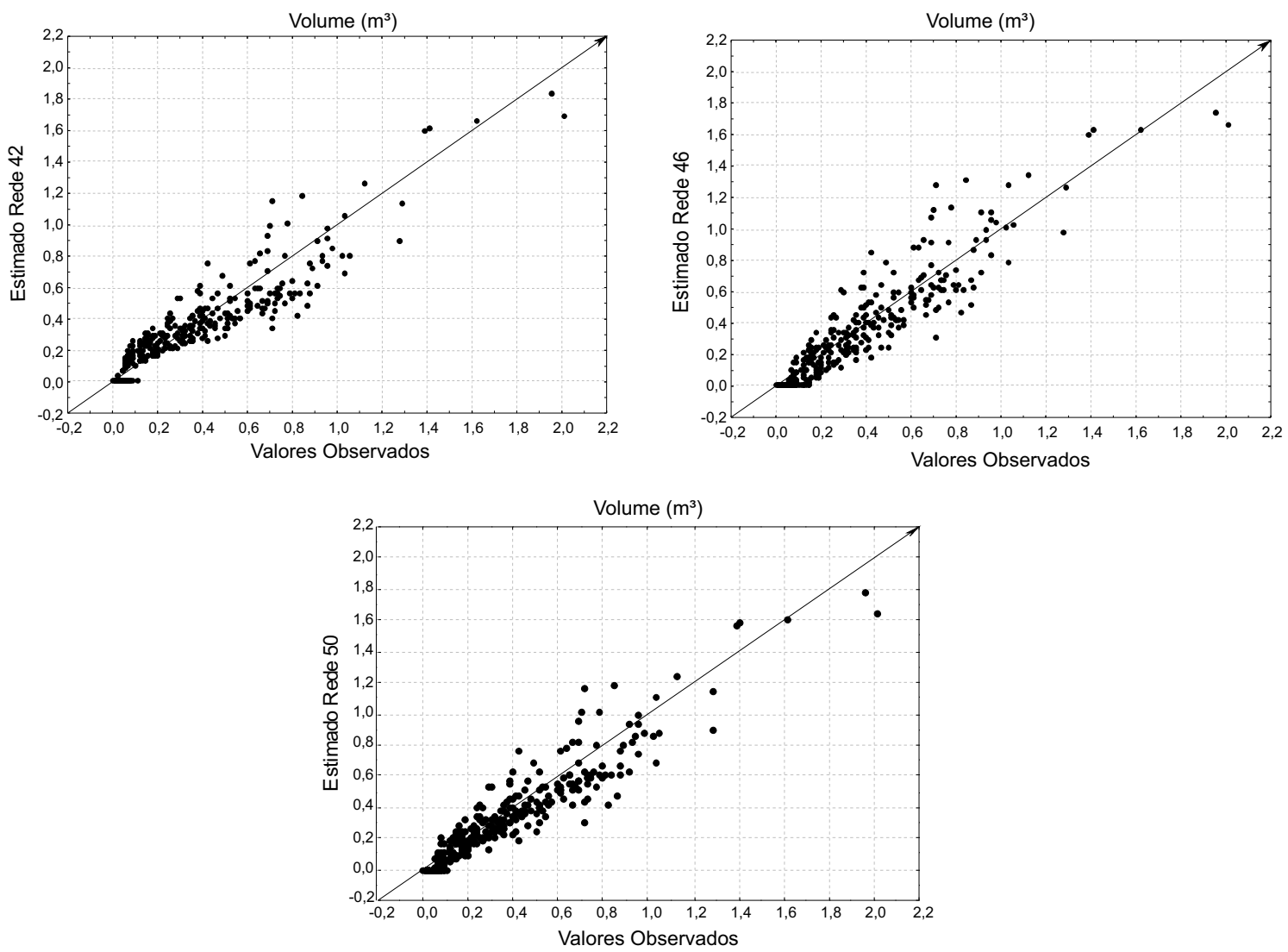

Figura 2 - Gráficos de valores observados versus valores estimados pelas redes neurais, que foram estatisticamente iguais aos valores observados.

Figure 2 - Observed versus predicted values by neural networks, wich were statistically similar to the observed values.

Revista Árvore, Viçosa-MG, v.38, n.2, p.289-295, 2014 


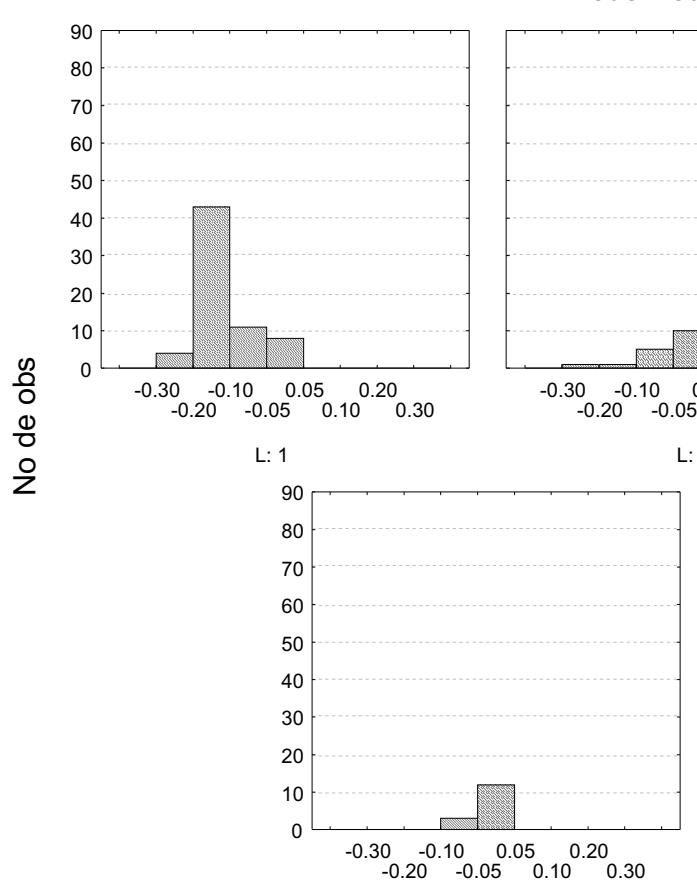

L: 4

\section{Rede Neural 46}

\section{Rede Neural 50}

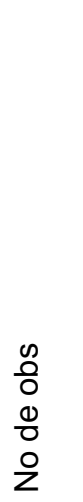

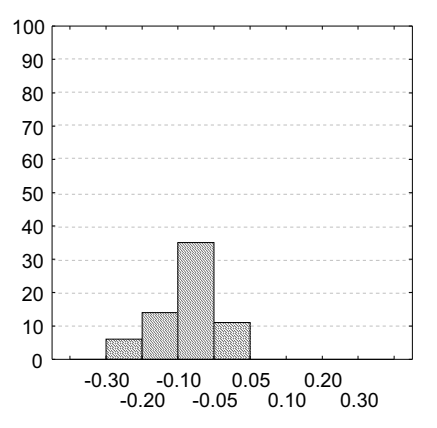

L: 1

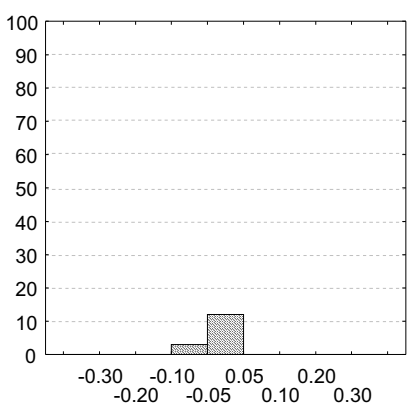

L: 4

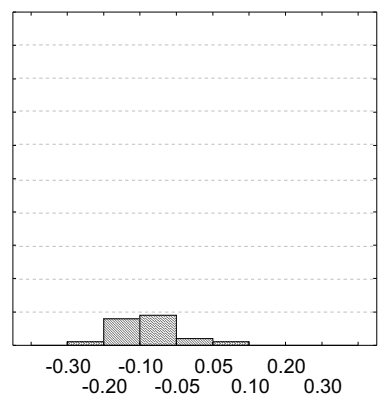

L: 2

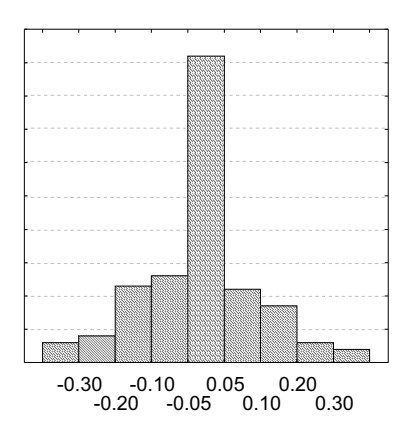

L: 5

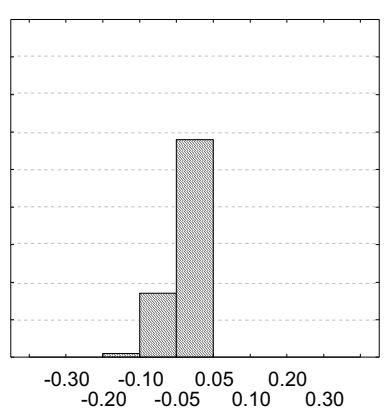

L: 3

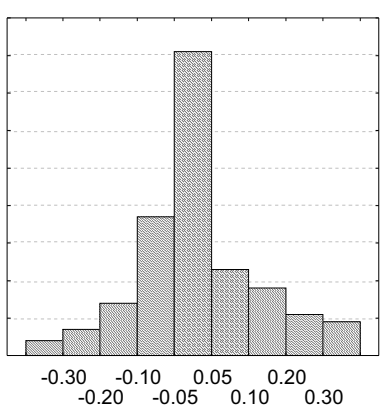

L: 5

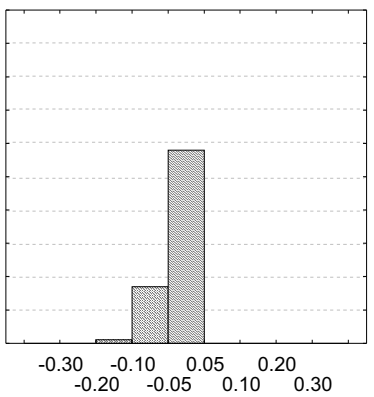

L: 3

Figura 3 - Distribuição dos resíduos das estimativas de volume geradas pelas redes 46 e 50, nos locais (L) 1 , 2 , 3 , 4 e 5 . Figure 3 - Residual distribuition for volume estimate by the networks 46 and 50, for each site. 


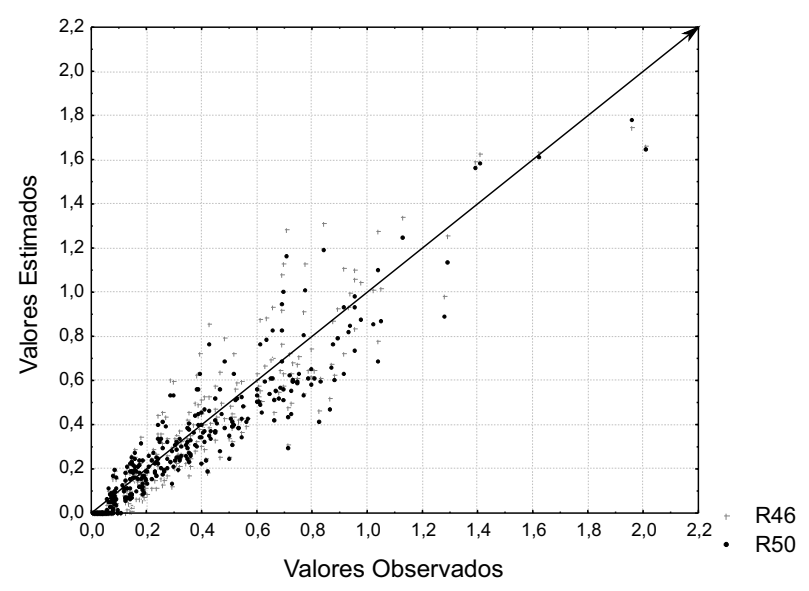

Figura 4-Gráficos dos valores observados e valores estimados pelas redes 46 e 50 .

Figure 4-Observed versus predicted scatterplot for the networks 46 and 50.

\section{DISCUSSÃO}

O uso de ferramentas de inteligência artificial na modelagem de crescimento e produção ainda é um assunto novo e pouco explorado. No entanto, esforços estão sendo feitos nessa direção, obtendo resultados promissores (GARBER; MAGUIRE, 2003; DIAMANTOPOULOU, 2005; GORGENS et al., 2009)

Os gráficos de resíduos mostram que, embora significativamente iguais pelo teste $t$, a rede 42 apresentou tendência de superestimar classes inferiores de volume (árvores de pequeno porte). Os gráficos de resíduos das redes 46 e 50 apresentaram comportamento adequado, sem tendenciosidade na estimativa.

As redes que apresentaram resultados coerentes, tanto no teste t quanto no gráfico de resíduos, foram a 46 e a 50 (Figuras 2, 3 e 4). Analisando a arquitetura, ambas possuíam 10 neurônios na primeira camada oculta, no entanto a rede 50 apresentou uma segunda camada com oito neurônios.

Com base nos resultados, verifica-se que a arquitetura 1 apresentou viés para classes de volume inferiores. As arquiteturas 2 e 3 apresentaram bons resultados, e a 3 exibiu resultados mais próximos ao observado.

Assim como em Gorgens et al. (2009), as redes apresentaram bons resultados para a estimativa de volumes de árvores individuais, em uma base de dados contendo diferentes locais e espécies. A arquitetura está diretamente relacionada com o poder de aprendizado da rede.

Assim, foi possível concluir que:

1. Para problemas de aproximação de funções de estimativa de volume de árvores, devem ser utilizadas redes com 10 ou mais neurônios na primeira camada intermediária.

2. Embora neste estudo o número de camadas não tenha influenciado estatisticamente o resultado, sugere-se a utilização de duas camadas, pois o “poder” de processamento de uma rede está relacionada à conectividade. No caso de trabalhos mais complexos, a demanda por camadas aumenta.

\section{REFERÊNCIAS}

BI, H.; LONG, Y. Flexible taper equation for sitespecific management of pinus radiata in New South Wales, Australia. Forest Ecology and Management, v.148, n.1, p.79-91, 2001.

CAMPOS, J. C. C.; LEITE, H. G. Mensuração florestal: perguntas e respostas. 2.ed. Viçosa, MG: Universidade Federal de Viçosa, 2006. 470p.

DIAMANTOPOULOU, M. J. Artificial neural networks as an alternative tool in pine bark volume estimation. Computers and Electronics in Agriculture, v.48, n.3, p.235-244, 2005

GARBER, S. M.; MAGUIRE, D. A. Modeling stem taper of three central oregon species using nonlinear mixed effects models and autoregressive error structures. Forest Ecology and Management, v.179, n.1, p.507-522, 2003.

GIMBLETT, H. R.; BALL, G. L. Neuronal network architectures for monitoring and simulating changes in forest resource management. AI Applications, v.9, n.2, p.103-123, 1995.

GORGENS, E. B. Estimação do volume de árvores utilizando redes neurais artificiais. 2006. 84f. Dissertação (Mestrado em Ciência Florestal) - Universidade Federal de Viçosa, Viçosa, MG, 2006.

GORGENS, E. B. et al. Estimação do volume de árvores utilizando redes neurais artificiais. Revista Árvore, v.33, n.6, p.1141-1147, 2009. 
HAYKIN, S. Redes neurais: princípios e prática. 2.ed. Porto Alegre: Bookman, 2001. 900p.

KAVZOGLU, T.; MATHER, P. M. The use of backpropagating artificial neural networks in land cover classification. International Journal of Remote Sensing, v.24, n.23, p.4907-4938, 2003.

LEITE, H. G.; GUIMARÃES, D. P.; CAMPOS, J. C. C. Descrição e emprego de um modelo para estimar múltiplos volumes de árvores. Revista Árvore, v.19, n.1, p.1-21, 1995.
SHARMA, M.; ODERWALD, R. G. Dimensionally compatible volume and taper equations.

Canadian Journal of Forest Research, v.31, n.5, p.797-803, 2001.

SILVA, G. F. et al. Análise da influência de diferentes tamanhos e composições de amostras no ajuste de uma relação hipsométrica para Eucalyptus grandis. Revista Árvore, v.31, n.4, p.685-694, 2007.

ZELL, A. et al. SNNS - Stuttgart Neural Network Simulator v.4.2. User manual, University of Stuttgart/University of Tübingen, 1998. 338 p. 
